\title{
Headache complaints associated with psychiatric comorbidity in a population-based sample
}

I.M. Benseñor ${ }^{1}$, L.F. Tófoli ${ }^{2}$ and L. Andrade 2
Departamentos de ${ }^{1}$ Clínica Médica, and ${ }^{2}$ Psiquiatria, Faculdade de Medicina, Universidade de São Paulo, São Paulo, SP, Brasil
Correspondence

I.M. Benseñor

Departamento de Clínica Médica

Hospital das Clínicas - PAMB

Av. Dr. Eneas C. de Aguiar, 155

05403-000 São Paulo, SP

Brasil

Fax: +55-11-3083-0827

E-mail: isabensenor@hcnet.usp.br

Research supported by FAPESP (No. 93/0501-4), and the Pro-Rector for Research, University of São Paulo. I.M. Benseñor was the recipient of a CNPq fellowship (2001-2002).

Received August 23, 2002 Accepted August 19, 2003

\begin{abstract}
The objective of the present study was to determine the frequency at which people complain of any type of headache, and its relationship with sociodemographic characteristics and psychiatric comorbidity in São Paulo, Brazil. A three-step cluster sampling method was used to select 1,464 subjects aged 18 years or older. They were mainly from families of middle and upper socioeconomic levels living in the catchment area of Instituto de Psiquiatria. However, this area also contains some slums and shantytowns. The subjects were interviewed using the Brazilian version of the Composite International Diagnostic Interview version 1.1. (CIDI 1.1) by a lay trained interviewer. Answers to CIDI 1.1 questions allowed us to classify people according to their psychiatric condition and their headaches based on their own ideas about the nature of their illness. The lifetime prevalence of "a lot of problems with" headache was $37.4 \%$ ( $76.2 \%$ of which were attributed to use of medicines, drugs/alcohol, physical illness or trauma, and $23.8 \%$ attributed to nervousness, tension or mental illness). The odds ratio (OR) for headache among participants with "nervousness, tension or mental illness" was elevated for depressive episodes (OR, 2.1; 95\%CI, 1.4-3.4), dysthymia (OR, 3.4; 95\% CI, 1.6-7.4) and generalized anxiety disorder (OR, 4.3; 95\% CI, 2.1-8.6), when compared with patients without headache. For "a lot of problems with" headaches attributed to medicines, drugs/alcohol, physical illness or trauma, the risk was also increased for dysthymia but not for generalized anxiety disorder. These data show a high association between headache and chronic psychiatric disorders in this Brazilian population sample.
\end{abstract}

Key words

- Headache

- Illness attribution

- Psychiatric comorbidity

- Brazil

- Odds ratio

\section{Introduction}

Headache is one of the most common complaints in the general population. It can be bothersome and interfere with routine activities and quality of life $(1,2)$. As is the case for some other high-prevalence pain symptoms, such as those involving the joints, chest, abdomen and back, headache is a complaint that remains poorly understood. In most cases, although extensively investigated by classical clinical and radiographic examinations, no organic cause is found. Some studies have shown that such pain syndromes are associated with common mental disorders and some personality traits $(1,3)$. However, the dilemma of whether psychopathology is the cause or a consequence of chronic 
pain is still a matter of controversy $(3,4)$.

There has been much discussion about a possible relationship between psychological factors and headache for a very long time. In 1937, Wolff(5) defined the "migraine personality" including ambition and perfectionism, mental instability and immaturity, vulnerability to frustrations, and shyness. Several studies at the community level have discussed the frequent association between headache of any type and psychiatric comorbidity $(1,6,7)$. A lot of data have correlated headache and personality traits, especially using patient samples from headache clinics $(3,4)$.

The psychiatric diagnoses most commonly associated with headaches are anxiety and depressive disorders (6,8-10), and the personality trait most frequently associated is neuroticism (2,11-13). Ossipova et al. (14) described two cases of migraine without aura, associated with panic attacks. They suggested that a pronounced autonomic dysregulation associated with psychological abnormalities could be related to both disorders, and that this comorbidity increases their severity. Marazziti et al. (15) found that this type of migraine is the most prevalent kind of headache among panic patients. Guidetti et al. (16), studying 100 patients from a Headache Center, found that from the youngest ages onwards, anxiety and depressive disorders represent a considerable clinical problem for both migraine and tension-type headache sufferers.

Population-based studies have confirmed this positive association in patient samples. In the Zurich Cohort Study of Young Adults (6) the combination of anxiety disorder and major depression was significantly associated with migraine. In a review paper, Merikangas and Stevens (10) discussed the importance of documenting an association between migraine and other comorbidities as the first step in creating a causal model. Accordingly, an index disease would cause or precipitate the manifestation of comorbid conditions (10). Breslau and Davis (11) and
Breslau et al. (12), studying a cohort of young adults, concluded that the presence of migraine increases the risk for depression and anxiety. Using Cox-proportional hazards, after 3.5 years of follow-up they found that both migraine and depression increased the risk for the first onset of each other. This favored an explanation of shared mechanisms. However, recent data from the Baltimore Epidemiological Catchment Area Follow-up Study (17) showed a strong crosssectional relationship between affective disorders and migraine headaches, but no association between previous affective disorder and incident cases of migraine headaches in a prospective manner.

There is little information about headache prevalence in Brazil. The only study presenting its association with psychiatric disorders was done on an adult sample (aged 18 years or over) in an urban area of Northeastern Brazil. An odds ratio (OR) of 4.4 was found for the association of headache symptoms and psychiatric comorbidity. Women were more at risk, and the prevalence increased with age (18). Migration, marital status, and low educational levels were associated with a higher frequency of headache. More recent data from a Brazilian sample of high-school students (aged 10-18 years) showed a lifetime prevalence of headache of $93.2 \%$, with a one-year prevalence of $82.9 \%$. Of these headaches, $72.8 \%$ were tensiontype and $9.9 \%$ migraine (19), according to the International Headache Society (IHS) criteria (20). Women had higher rates than men. Sanvito et al. (21) interviewed 595 medical students. When classifying their headaches according to IHS criteria, they observed that the one-year prevalence for any kind of headache was $40 \%$ and that $40.2 \%$ of those were migraine. The migraine prevalence was $54 \%$ for women and $28.3 \%$ for men, and was considered incapacitating by half of the sufferers. No information about psychiatric comorbidity or personality traits was available in these last two studies. 
We analyzed data from a populationbased catchment area mental health survey in the city of São Paulo, Brazil, to determine the prevalence of people complaining of "a lot of problems with" any kind of headache, the relationship with sociodemographic characteristics and the co-occurrence of psychiatric disorders.

\section{Material and Methods}

The study population lived in the catchment area of the University of São Paulo Medical Center, a 2000-bed tertiary care facility in São Paulo, the largest city in South America and one of the largest cities in the world (ten million inhabitants). The catchment area included two districts of the city covering a geographic area of $10.5 \mathrm{~km}^{2}$. The population in this area consists mainly of families of middle and upper socioeconomic levels. However, this area also contains some slums and shantytowns.

In order to improve the probability of observing young psychotics and old-age psychiatric morbidity, all persons aged 18-24 years and 60 years or older, living in each selected household, were interviewed with a probability equal to one. Of the remaining individuals aged 25-29 years living in the selected household, one was chosen for interview based on the Kish and Frankel selection table $(22,23)$.

From the 950 households, 1,906 people were selected to participate in the study based on the three age strata described above. Of these, 442 refused to participate, resulting in a final sample of 1,464 subjects, with an individual response of $76.8 \%$.

\section{Psychiatric assessment}

The psychiatric diagnoses were based on face-to-face interviews. The instrument used was the Brazilian version of the Composite International Diagnostic Interview (CIDI) (24), version 1.1. This is a structured psychi- atric interview (25) designed for use by lay interviewers. The instrument provides lifetime, 12- and 1-month prevalence estimates for ICD-10 diagnoses (International Classification of Disease-Related Health Problems, 10th revision, WHO, 1992) (26). For this study, we tested the association of lifetime psychiatric disorders that could be related to headache: depression, anxiety, alcohol and nicotine dependence, somatization, and bulimia.

\section{Headache diagnosis}

The CIDI 1.1 asks whether the person has experienced "a lot of problems with" headache during his/her lifetime and/or has taken any medication for headache, three or more times during a week. Answers to these questions were classified as: i) negative answer; ii) positive answer, but patient did not seek medical help because of symptoms, did not take any medication, or symptoms did not interfere with routine activities; iii) positive answer, but symptoms were caused by medicines, drugs or alcohol; iv) positive answer, but symptoms were the consequence of physical illness or trauma; v) positive answer, but symptoms were the consequence of nervousness, tension, anxiety, depression, mental illness, or no defined diagnosis. Thus, the prevalence rate does not reflect the actual prevalence of headache, but is restricted to people with a lot of problems with headaches (a small portion of all the people with headaches). Data from the CIDI did not allow us to classify headaches according to the IHS criteria (20), but it was possible to consider answers 3 and 4 as headaches attributed by the patient to the use of medication, drugs or alcohol, physical illness or trauma, and answer number 5 as potentially associated with psychiatric disorders. The attributed cause was classified into two categories: lifestyle and/or physical conditions were considered to be the cause of answers 3 or 4 , and psychological conditions were consid- 
ered to be the cause of answer 5 , as used previously by Nimnuan et al. (27).

\section{Sociodemographic measurements}

We considered the effects of several sociodemographic variables such as age, gender, marital status, and educational level (years of education). Age was coded by category, as ranges 18-24, 25-34, 35-44, 45-54, 55-64, and 65 and over. Gender was coded

Table 1. Lifetime prevalence rates for "a lot of problems with" headache of any type, headaches attributed to lifestyle and/or physical conditions, and headaches attributed to psychological conditions, by gender and age group.

\begin{tabular}{|c|c|c|}
\hline & Men & Women \\
\hline \multicolumn{3}{|l|}{18 to 24 years } \\
\hline N & 109 & 225 \\
\hline Any headache & $19.3(3.3)$ & $37.3(2.6)$ \\
\hline Lifestyle headaches & $8.2(2.2)$ & $21.3(2.0)$ \\
\hline Psychological headaches & $6.4(2.4)$ & $6.7(1.4)$ \\
\hline \multicolumn{3}{|l|}{25 to 34 years } \\
\hline $\mathrm{N}$ & 83 & 166 \\
\hline Any headache & $23.1(4.8)$ & $54.0(4.3)$ \\
\hline Lifestyle headaches & $10.5(3.0)$ & $22.7(2.5)$ \\
\hline Psychological headaches & $7.0(2.6)$ & $17.3(3.1)$ \\
\hline \multicolumn{3}{|l|}{35 to 44 years } \\
\hline $\mathrm{N}$ & 73 & 181 \\
\hline Any headache & $20.3(5.1)$ & $48.0(4.0)$ \\
\hline Lifestyle headaches & $10.9(3.8)$ & $32.5(3.4)$ \\
\hline Psychological headaches & $4.3(2.4)$ & $10.4(2.0)$ \\
\hline \multicolumn{3}{|l|}{45 to 54 years } \\
\hline $\mathrm{N}$ & 72 & 119 \\
\hline Any headache & $30.8(5.2)$ & $48.8(4.5)$ \\
\hline Lifestyle headaches & $11.7(3.7)$ & $33.0(4.0)$ \\
\hline Psychological headaches & $14.5(4.1)$ & $8.1(2.8)$ \\
\hline \multicolumn{3}{|l|}{55 to 64 years } \\
\hline $\mathrm{N}$ & 47 & 105 \\
\hline Any headache & $38.4(8.5)$ & $56.4(5.1)$ \\
\hline Lifestyle headaches & $30.0(7.6)$ & $41.3(5.7)$ \\
\hline Psychological headaches & $5.0(3.5)$ & $5.8(2.5)$ \\
\hline \multicolumn{3}{|l|}{65 or older } \\
\hline $\mathrm{N}$ & 98 & 185 \\
\hline Any headache & $19.3(4.7)$ & $38.4(3.0)$ \\
\hline Lifestyle headaches & $12.1(3.6)$ & $24.3(2.4)$ \\
\hline Psychological headaches & $3.1(1.7)$ & 7.6 (1.9) \\
\hline
\end{tabular}

Data are reported as percent (SEM). Lifestyle headaches are headaches attributed to lifestyle and/or physical conditions; psychological headaches are headaches attributed to psychological conditions. $\mathrm{N}=$ Number of subjects. dichotomously (male, female). Marital status was coded as married or not married. Years of education were coded by category as ranges $0-8,9-11,12-15$, and 16 or more.

\section{Statistical analysis}

Since the data in this report were obtained from a complex stratified sample, they were weighted for differential probabilities of selection and nonresponse.

A post-stratification of known population characteristics of sex and regional geographic groupings in the sample age range was also made in order to compensate for discrepancies between the sample and the original census population data. A matrix with the following factors was constructed to calculate the final weighting: age, sex, age strata, number of persons in each stratum by household, and a post-stratification factor.

Logistic regression analysis was used to examine the association between demographic factors, psychiatric disorders and the headache symptom. As a result of the complex sample design and weighting, estimates of standard errors based on the usual assumption of equal-probability simple random samples become biased. Thus, standard errors for prevalence and logistic regression coefficients were computed using the jackknife repeated replications method, to adjust for the design effects introduced by the clustering and weighting of observations $(22,23)$. All evaluations of significance were based on two-sided tests using 0.05 as the level of significance.

\section{Results}

The lifetime prevalence for "a lot of problems with" headache of any type for the total sample was $37.4 \%$. Of this, $76.2 \%$ was attributed to medicines or drugs/alcohol and to physical illness or trauma (cause attributed to lifestyle and/or physical conditions), and $23.8 \%$ was attributed to nervousness, ten- 
sion or mental illness (cause attributed to psychological conditions). Table 1 shows the lifetime prevalence rates for "a lot of problems with" headache of any type, headache attributed to lifestyle and/or physical conditions, and headache attributed to psychological conditions by gender and age stratum. The lifetime rates for "a lot of problems with" any kind of headache ranged from $19.3 \%$ (for men aged 65 years or older) to $56.4 \%$ (for women aged 55-64 years), showing that this is a very common symptom. The lifetime frequency of "a lot of problems with" headache attributed to lifestyle and/or physical conditions was higher than the lifetime frequency of "a lot of problems with" headache attributed to psychological conditions, for all ages. This increased with age for men and women up to the age of 64 years (peaking between 55 and 64 years). After this age, we observed a decline for both genders. The frequency of "a lot of problems with" headaches attributed to psychological conditions was higher in women of all ages, except in the 45-54-year age stratum. The lifetime prevalence of "a lot of problems with" headaches attributed to psychological conditions reached a peak in both genders in the age stratum of 25-34 years. After the age of 54 years, the frequency decreased to levels that were similar to those of the 18-24year age group.

Table 2 shows the OR (controlled for age and gender) for any kind of headache, headache attributed to lifestyle and/or physical conditions, and headache attributed to psychological conditions according to sociodemographic characteristics of the study population. Gender had a major effect, with women being 1.5 to 2.9 times more at risk than men. As we are reporting the lifetime prevalence of symptoms, a pattern of increasing prevalence with age would be expected. This was the case for "a lot of problems with" headaches attributed to lifestyle and/or physical conditions, in which the risk increased with age up to the 55-64-year age stratum and decreased thereafter. This was not the case for "a lot of problems with" headaches attributed to psychological conditions, for which the risk was higher only in the 25-34-year age stratum (OR, 2.1; 95\% CI, 1.1-4.0), indicating that this age group was at major risk for the disorders grouped under this heading. There was no effect of years of

Table 2. Association between headache, sociodemographic measures and psychiatric comorbidity.

\begin{tabular}{|c|c|c|c|}
\hline & $\begin{array}{c}\text { Many problems } \\
\text { with any kind of } \\
\text { headache } \\
(\mathrm{OR}, 95 \% \mathrm{Cl})\end{array}$ & $\begin{array}{c}\text { Many problems } \\
\text { with lifestyle } \\
\text { headaches } \\
\text { (OR, } 95 \% \mathrm{Cl})\end{array}$ & $\begin{array}{c}\text { Many problems } \\
\text { with psychological } \\
\text { headaches } \\
(\mathrm{OR}, 95 \% \mathrm{Cl})\end{array}$ \\
\hline \multicolumn{4}{|l|}{ Gender } \\
\hline Men & 1.0 (reference) & 1.0 (reference) & 1.0 (reference) \\
\hline Women & $2.9(2.1-1.4)$ & $2.9(2.0-4.1)$ & $1.5(1.0-2.3)$ \\
\hline \multicolumn{4}{|l|}{ Age strata (years) } \\
\hline $18-24$ & 1.0 (reference) & 1.0 (reference) & 1.0 (reference) \\
\hline $25-34$ & $1.7(1.2-2.3)$ & $1.1(0.7-1.7)$ & $2.1(1.1-4.0)$ \\
\hline $35-44$ & $1.3(0.9-1.9)$ & $1.6(1.1-2.4)$ & $1.2(0.6-2.2)$ \\
\hline $45-54$ & $1.7(1.2-2.5)$ & $1.7(1.1-2.5)$ & $1.8(0.8-3.9)$ \\
\hline $55-64$ & $2.3(1.5-3.6)$ & $3.1(2.0-4.8)$ & $0.8(0.3-2.1)$ \\
\hline$\geq 65$ & $1.0(0.7-1.4)$ & $1.2(0.8-1.8)$ & $0.9(0.4-1.7)$ \\
\hline \multicolumn{4}{|l|}{ Years of education } \\
\hline $0-8$ & $1.3(0.9-1.8)$ & $1.0(0.8-1.4)$ & $0.9(0.5-1.4)$ \\
\hline $9-11$ & $0.9(0.7-1.3)$ & $0.8(0.5-1.1)$ & $0.9(0.5-1.7)$ \\
\hline $12-15$ & $0.8(0.6-1.2)$ & $1.1(0.7-1.6)$ & $0.6(0.3-1.1)$ \\
\hline$\geq 16$ & 1.0 (reference) & 1.0 (reference) & 1.0 (reference) \\
\hline \multicolumn{4}{|l|}{ Marital status } \\
\hline Alone & $1.0(0.7-1.3)$ & $0.9(0.7-1.3)$ & $1.0(0.7-1.5)$ \\
\hline Together & 1.0 (reference) & 1.0 (reference) & 1.0 (reference) \\
\hline \multicolumn{4}{|l|}{ Comorbidity } \\
\hline Depression & $1.8(1.3-2.4)$ & $1.3(1.0-1.8)$ & $2.1(1.3-3.4)$ \\
\hline Depressive episode & $1.9(1.4-2.5)$ & $1.4(1.0-1.9)$ & $2.1(1.4-3.4)$ \\
\hline Dysthymia & $4.2(2.3-7.6)$ & $2.2(1.1-4.3)$ & $3.4(1.6-7.4)$ \\
\hline Any anxiety disorder & $2.3(1.6-3.4)$ & $1.1(0.7-1.7)$ & $3.0(1.9-4.7)$ \\
\hline Panic disorder & $1.7(0.8-3.7)$ & $1.1(0.5-2.4)$ & $1.9(0.9-3.9)$ \\
\hline GAD & $3.2(1.5-6.9)$ & $1.3(0.7-2.5)$ & $4.3(2.1-8.6)$ \\
\hline Any phobia & $2.0(1.4-2.8)$ & $1.2(0.7-1.9)$ & $1.9(1.0-3.6)$ \\
\hline Somatoform disorder & $5.0(2.9-8.4)$ & $1.0(0.5-1.8)$ & $11.0(6.1-19.6)$ \\
\hline \multicolumn{4}{|l|}{ Dependence } \\
\hline Alcohol & $1.5(0.8-2.7)$ & $1.0(0.5-1.9)$ & $1.2(0.5-3.0)$ \\
\hline Nicotine & $1.2(0.9-1.6)$ & $1.3(1.0-1.8)$ & $0.9(0.5-1.6)$ \\
\hline Bulimia nervosa & $0.8(0.3-2.0)$ & $0.5(0.1-2.1)$ & $0.7(0.1-7.6)$ \\
\hline No psychiatric disorder & 1.0 (reference) & 1.0 (reference) & 1.0 (reference) \\
\hline
\end{tabular}

$\mathrm{OR}=$ odds ratio; $95 \% \mathrm{Cl}=95 \%$ confidence interval; $\mathrm{GAD}=$ generalized anxiety disorder. Lifestyle headaches are headaches attributed to lifestyle and/or physical conditions; psychological headaches are headaches attributed to psychological conditions. 
education or marital status on the risk for "a lot of problems with" headache.

Table 2 also shows the OR for the association of headaches and psychiatric comorbidity. The OR for "a lot of problems with" any kind of headache associated with depression was 1.8 (95\%CI, 1.3-2.4). However, when we subdivided depression into depressive episode and dysthymia, most of the comorbidity was concentrated in dysthymia (OR, 4.2; 95\% CI, 2.3-7.6), in comparison with depressive episodes (OR, 1.9; 95\%CI, 1.4-2.5). For anxiety (OR, 2.3; $95 \% \mathrm{CI}, 1.6-3.4)$, the association was again higher for generalized anxiety disorder (OR, $3.2 ; 95 \% \mathrm{CI}, 1.5-6.9)$, which is a more chronic disturbance, in comparison with panic disorder (OR, 1.7; 95\%CI, 0.8-3.7) or any kind of phobias (OR, 2.0; 95\%CI, 1.4-2.8). The same pattern was found for "a lot of problems with" headaches attributed to psychological conditions, with slight differences in the OR. For "a lot of problems with" headaches attributed to lifestyle and/or physical conditions, the risk of psychiatric comorbidity was increased for dysthymia (OR, 2.2; 95\%CI, 1.1-4.3) but not for generalized anxiety disorder (OR, 1.3; 95\%CI, 0.7-2.5). As expected, the OR for association of somatoform disorders and "a lot of problems with" headaches attributed to psychological conditions was very high (OR, $11 ; 95 \% \mathrm{CI}$, 6.1-19.6) because headache due to these conditions could be part of the diagnostic criteria for somatoform disorders. In our sample, for people with somatoform disorders, the most common physical symptoms in order of frequency were headache (44\%), followed by chest pain $(17.9 \%)$, abdominal pain (17.9\%), and back pain (16.7\%). There was no association between headache and alcohol or nicotine dependence, or bulimia.

\section{Discussion}

In this cross-sectional study conducted on Brazilian adults, we detected an associa- tion between headache and psychiatric disorders. "A lot of problems with" headache is a very prevalent symptom in the general population. At least one in every three persons has had such symptoms at some time in their lives. Because we only assessed headaches of some sort of severity, it is quite probable that these numbers are an underestimate of the actual prevalence of headache in this community. Systematic comparison with previous population-based studies is difficult because of different instruments used to assess headache. Nonetheless, our finding of a $37.4 \%$ frequency of lifetime headache is far below the nearly $80 \%$ frequency of tension-type headache reported by Rasmussen in Sweden (2) and the $72.8 \%$ value reported by Barea et al. (19) for children and adolescents in Brazil, using the IHS criteria. However, our rate is slightly above the lifetime prevalence of $25.9 \%$ of headache complaints reported by Kroenke and Price (1) in the Epidemiologic Catchment Area Program study.

As in other studies conducted on the general population $(2,18)$, headache symptoms are both age and gender dependent. "A lot of problems with" headache is more common among women. Headaches that are symptomatic (cause attributed to lifestyle and/or physical conditions, such as headache due to fever, hangover and other lifestyle causes, clinical or neurological disorders) increased with age. The higher prevalence of headaches attributed to nervousness or mental illness in the 25-34-year age stratum may indicate that young age cohorts more frequently experience these types of symptoms. The lack of association of headache symptoms with educational level and marital status also agrees with other population-based studies.

In agreement with previous studies $(1,9$, $18,19,27)$, one of which was conducted on a Brazilian population (18), there was a high comorbidity among people complaining of "a lot of problems with" headache, espe- 
cially headaches attributed to nervousness or mental illness with depression and anxiety disorders. People with "a lot of problems" with headache have at least a two-fold increased likelihood of having lifetime anxiety or depressive disorder. This association is especially strong with chronic disorders like generalized anxiety disorder and dysthymia. This is in agreement with data from Italian patients with episodic and chronic tension-type headache, described by Guidetti et al. (16). The most common anxiety disorder diagnosed was generalized anxiety disorder $(44.7 \%)$, and the most common depression disorder was dysthymia $(16.6 \%)$. These kinds of findings are still scarcely available in the literature because most studies do not subdivide depressive and anxiety disorders into subgroups. There was no association of headache and panic disorder in our sample. However, the lack of significance of this result may have been due to the low prevalence $(1.5 \%)$ of panic disorder in our sample.

The present study has several limitations. First, we did not evaluate the real prevalence of headache in the population sample but the prevalence of "a lot of problems with" headache. Therefore, our data give an underestimate of the real prevalence of headache in the population. Data from a population-based telephone interview survey in Maryland, USA, which included residents 12-29 years of age who reported having had a headache during the previous year, showed that only $26.7 \%$ of women and $13.6 \%$ of men had ever sought a physician because of the headache problem (28). Most people with headache have episodic tension-type headache, which is sporadic and of mild intensity and have never sought a physician for medical advice about a headache problem. So again, when we asked about "many problems with headaches", these people were probably not included and underestimation was again possible.

Second, we did not have the information to classify headache subtypes according to the IHS criteria. However, it is possible to obtain the individual attributed causes of headache by using the answers to the CIDI questions. We were probably preferentially selecting mostly the headaches attributable to psychological conditions as primary tension-type and migraine headaches with no alteration upon physical examination. Most of the headaches attributable to lifestyle and/ or physical conditions (those due to medicines, drugs and alcohol, clinical and neurological diseases) would then have been selected as secondary headaches. However, since the IHS criteria were not available in this sample, some kind of misclassification was very likely. This may have influenced our results showing a positive association of headache attributed to lifestyle and/or physical conditions with dysthymia. This would be reflected in some of the primary headaches (migraine or tension-type) being erroneously identified as secondary headaches.

According to our definition, headaches due to nervousness or mental sickness were more prevalent between 25 and 44 years of age, an age stratum in which primary headaches are really more prevalent. The clearly greater association between some psychiatric disorders and "primary headaches" is another point that suggests that we made a true assumption in classifying most primary headaches into the group of headaches attributed to psychological conditions. This agrees with previous observations in the literature $(5,16,17,19,28,29)$.

The strength of the present study is that the information came from a general population sample and was collected using standardized interviews to assess psychiatric morbidity. Few studies in the literature have provided data on the relationship between headache and other psychiatric disorders like bulimia, nicotine and alcohol dependence. Most of the available data are about anxiety and depression as a whole. Only a small number of studies have included the sub- 
types of anxiety (panic disorder, generalized anxiety disorders and phobias) and depression (dysthymia, depressive episode) (30).

The results of the present study are not representative of the entire Brazilian population because of the wide variety of cultural and socioeconomic influences in the country.

It is clear from our data that headache is correlated with common chronic psychiatric disorders such as generalized anxiety disorders, among anxiety disorders, and dysthymia among depression disorders. There is also a very strong comorbidity between headache and somatoform disorders. Additional studies are needed for a better understanding of the relationships between these disorders.

\section{References}

1. Kroenke K \& Price RK (1993). Symptoms in the community: prevalence, classification, and psychiatric comorbidity. Archives of Internal Medicine, 153: 2474-2480.

2. Rasmussen BK (1995). Epidemiology of headache. Cephalalgia, 15: 45-68.

3. Mongine F, Ferla E \& Maccagnani C (1992). MMPI profiles in patients with headache or craniofacial pain: a comparative study. Cephalalgia, 12: 91-98.

4. Mongine F, Defilippi N \& Negro C (1997). Chronic daily headache. A clinical and psychological profile before and after treatment. Headache, 37: 83-87.

5. Wolff HG (1937). Personality features and reactions of subjects with migraine. Archives of Neurology and Psychiatry, 37: 895-921.

6. Merikangas KR, Angst J \& Isler H (1990). Migraine and psychopathology: results of the Zurich Cohort Study of Young Adults. Archives of General Psychiatry, 47: 849-853.

7. Breslau N, Davis GC \& Andreski P (1991). Migraine, psychiatric disorders and suicide attempts: an epidemiological study of young adults. Psychiatry Research, 37: 11-23.

8. Mongine F, Ibertis F \& Ferla E (1994). Personality characteristics before and after treatment of different head pain syndromes. Cephalalgia, 14: 368-373.

9. Mitsikostas DD \& Thomas AM (1999). Comorbidity of headache and depressive disorders. Cephalalgia, 19: 211-217.

10. Merikangas KR \& Stevens DE (1997). Comorbidity of migraine and psychiatric disorders. Neurologic Clinics, 15: 115-123.

11. Breslau N \& Davis GC (1992). Migraine, major depression and panic disorder: a prospective epidemiological study of young adults. Cephalalgia, 12: 85-90

12. Breslau N, Davis GC, Schultz LR \& Peterson EL (1994). Migraine and major depression: a longitudinal study. Headache, 34: 387-393.

13. Ziegler DK \& Paolo AM (1995). Headache symptoms and psychological profile of headache prone individuals. A comparison of clinic patients and controls. Archives of Neurology, 52: 602-606.

14. Ossipova VV, Kolosova OA \& Vein AM (1999). Migraine associated with panic attacks. Cephalalgia, 19: 728-731.

15. Marazziti D, Toni C, Pedri S, Bonuccelli U, Pavese N, Lucetti C, Nuti A, Muratorio A \& Cassano GB (1999). Prevalence of headache syndromes in panic disorder. International Clinical Psychopharmacology, 14: 247-251.

16. Guidetti V, Fabrizi P, Giannantoni AS, Napoli L, Bruni O \& Trillo S (1998). Headache and psychiatric comorbidity: clinical aspects and outcome in an 8-year follow-up study. Cephalalgia, 18: 455-462.

17. Swartz KL, Pratt LA, Armenian HK, Ching Lee MS \& Eaton WW
(2000). Mental disorders and the incidence of migraine headaches in a community sample. Archives of General Psychiatry, 57: 945-950.

18. Bastos SB, Almeida-Filho N \& Santana VS (1993). Prevalence of headache as a symptom in the urban area of Salvador, Bahia, Brazil. Arquivos Brasileiros de Neuropsiquiatria, 51: 307-312.

19. Barea LM, Tannhauser M \& Rotta NT (1996). An epidemiological study of headache among children and adolescents in southern Brazil. Cephalalgia, 16: 545-549.

20. International Headache Society (1988). Classification and diagnostic criteria for headache disorders, cranial neuralgias and facial pain. Headache Classification Committee of the International Headache Society. Cephalalgia, 8 (Suppl 7): 1-96

21. Sanvito WL, Monzillo PH, Peres MF, Martinelli MO, Fera MP, Gouveia DA, Murachovsky J, Salomão WR \& Leme RJ (1996). The epidemiology of headache in medical students. Headache, 36: 316319.

22. Kish L (1965). Survey Sampling. John Wiley \& Sons, New York.

23. Kish L \& Frankel MR (1970). Balanced repeated replications for standard errors. Journal of the American Medical Association, 65: 1071-1094.

24. Miranda CT, Mari JJ, Ricciardi A \& Arruda ME (1990). Patients' reactions to CIDI in Brazil. In: Stefanis CN, Rabavillas AD \& Soldatos CR (Editors), Psychiatry: A World in Perspective. Elsevier, Amsterdam, The Netherlands, 133-137.

25. Lopes CS (1994). Reliability of the Brazilian version of the CIDI in a case-control study of risk factors for drug abuse among adults in Rio de Janeiro. Bulletin of the Pan-American Health Organization, 28: 34-41.

26. World Health Organization (1992). Mental health and behavioral disorders. In: International Classification of Diseases. 10th revision. World Health Organization, Geneva, Switzerland, 311-387.

27. Nimnuan C, Hotopf M \& Wessely S (2001). Medically unexplained symptoms. An epidemiological study in seven specialties. Journal of Psychosomatic Research, 51: 361-367.

28. Breslau N (1998). Psychiatric comorbidity in migraine. Cephalalgia, 18 (Suppl 22): 56-61.

29. Linet MS, Celentano DD \& Stewart WF (1991). Headache characteristics associated with physician consultation: a population-based survey. Annals of Preventive Medicine, 7: 40-46.

30. The Italian Collaborative Group for the Study of Psychopathological Factors in Primary Headaches (1999). Psychiatric comorbidity and psychosocial stress in patients with tension-type headache from headache centers in Italy. Cephalalgia, 19: 159-164. 